\title{
Contexts of HIV-Related Risk Behaviors among Male Customers at Asian Massage Parlors in San Francisco
}

\author{
Tooru Nemoto, ${ }^{1}$ Mariko Iwamoto, ${ }^{1}$ Elnaz Eilkhani, ${ }^{1}$ Maria Sakata, \\ Mai Nhung Le, ${ }^{2}$ and Anne Morris ${ }^{3}$ \\ ${ }^{1}$ Public Health Institute, 555 12th Street, Suite 290, Oakland, CA 94607, USA \\ ${ }^{2}$ San Francisco State University, San Francisco, CA, USA \\ ${ }^{3}$ Walden University, Minneapolis, MN, USA \\ Correspondence should be addressed to Tooru Nemoto; tnemoto@phi.org
}

Received 7 November 2013; Accepted 14 January 2014; Published 4 March 2014

Academic Editors: B. Best and F. Uribe-Salas

Copyright (C) 2014 Tooru Nemoto et al. This is an open access article distributed under the Creative Commons Attribution License, which permits unrestricted use, distribution, and reproduction in any medium, provided the original work is properly cited.

\begin{abstract}
Based on focus groups for male customers who frequented Asian massage parlors in San Francisco, the present study described their sexual and drug use behaviors and attitudes toward practicing safe sex with Asian masseuses. A pervasive view among patrons was that they could engage in sex with masseuses without using a condom if they offered extra money. Their sexual behaviors with Asian masseuses were influenced by perceptions about vulnerability toward HIV/STIs, substance use behaviors, and masseuses' initiation of condom use, which was often governed by unspoken rules at parlors. Customers perceived massage parlors as being a safe place compared with street sex venues. Some customers sought emotional attachment with Asian masseuses and expressed stereotypical views toward them as being docile and submissive. Culturally appropriate HIV/STI prevention programs (e.g., communication about and practicing 100\% condom use) are needed to target both customers and Asian masseuses at multiple levels (individual, owner/manager, work environment, and community levels). Also, customers' and masseuses' perceptions toward relationships at massage parlors and power dynamics need further investigation to promote safe work environments and $100 \%$ condom use at massage parlors.
\end{abstract}

\section{Introduction}

Prostitution is illegal in San Francisco and other large cities in the USA; however, male customers are able to negotiate with female sex workers (FSWs)/masseuses for sexual services at massage parlors. Commercial sex venues at massage parlors in the USA are distinguished by the service providers (mostly Asian immigrant women from South Korea, China, Vietnam, Thailand, and other Asian countries) and service delivery systems which are based on private negotiations between Asian masseuses and customers to exchange money for sexual services. Typically, customers pay massage fees at massage parlor premises and enter into a massage room and negotiate sexual services. A health promotion study for Asian masseuses in San Francisco was implemented in 2002 [1-3]. The study identified 23 "Asian" massage parlors in downtown San Francisco. Based on the baseline data collected from 105 Asian masseuse participants, $11 \%$ and $19 \%$ of the participants reported having engaged in unprotected vaginal and oral sex with customers during the past 30 days, respectively [13]. The qualitative study showed that Asian masseuses face difficult choices over condom use due to economic incentives to earn extra money through not using a condom, as well as keeping relationships with regular customers [2]. In addition, intimidation, such as fear of threats and victimization from massage parlor managers and customers, has contributed to unsafe sexual practices among Asian masseuses [2]. However, little is known about HIV-related risk behaviors and the environmental and cognitive factors influencing the risk behaviors among male customers at massage parlors in San Francisco and elsewhere in the USA, despite the fact that a number of "Asian" massage parlors are operating in almost all major US cities.

In developing countries, particularly in sub-Saharan Africa and Asia, FSWs are considered to be a "bridge" population for HIV transmission to the general population 
[4-6]. These HIV prevention studies that have focused on FSW and examined male customers' condom use and other risk behaviors and factors $[7,8]$, such as STI prevalence $[9,10]$, HIV testing $[11,12]$, substance use $[10,13]$, HIV knowledge [10, 14], susceptibility toward HIV infections $[15,16]$, and reporting multiple sex partners $[10,11]$. Male customers' inconsistent condom use was correlated with negative attitudes toward female sex workers and condom use, lower levels of education, AIDS knowledge, and selfefficacy, younger age, and being a regular customer and frequent user of sexual services $[10,14]$.

Most of these studies investigating HIV-related risks among male customers have been conducted outside of the USA, despite the large number of men that pay for sex with FSWs who work on the street, at massage parlors, bars/clubs, and strip joints and through escort or dating services by phone or the Internet [17]. A study conducted in Atlanta, Georgia, revealed that male customers who paid for sex with street female sex workers were frequently observed to have a history of crack use (23\%) and injection drug use (11\%) and to be infected with HIV (3\%), Syphilis (10\%), and Hepatitis B (25\%) [18]. FSWs working at brothels in Nevada were likely to report that male customers were often unwilling to use condoms, although customers were aware of the mandatory condom use law in Nevada [19]. More than half of the sex workers in this study reported having had at least one customer who resisted using a condom in the previous month. There is a huge gap in HIV prevention literature regarding HIV-related risk behaviors and the sociocultural contexts of engaging in sex with Asian FSWs at massage parlors in the USA.

This study aimed to describe HIV-related risk behaviors, focusing on unprotected sex with Asian masseuses and sex under the influence of alcohol and illicit drugs among customers who frequented massage parlors. The study also aimed to understand in what ways the following factors influenced customers' risky sexual behaviors: (1) perception of risk for HIV/STIs, (2) attitudes toward Asian massage parlors and masseuses, (3) perception of rules and regulations of condom use at massage parlors, and (4) perception of control over safe sex negotiation with Asian masseuses. The study provided practical suggestions for future HIV/STI prevention programs and public policy changes for promoting health for both Asian masseuses and their customers.

\section{Method}

2.1. Procedure. The present study is part of a larger HIV prevention intervention study for Asian masseuses in San Francisco. First, we conducted mapping and identified a total of 23 massage parlors in the downtown area of San Francisco where Asian masseuses exchanged sex for money with customers. Massage business establishments that provided physical relaxation massage were excluded from the study.

Data reported here were collected from focus groups for male customers that frequented massage parlors in San Francisco. Participants in the focus groups were recruited through referrals from masseuses and recruitment fliers which were distributed near the targeted massage parlors. The study had two waves of data collection. In 2002, three focus groups for male customers were conducted specific to racial/ethnic groups: Caucasian $(n=4)$, African-American $(n=5)$, and Asian ( $n=9)$. In 2006, two focus groups, mixing participants with different racial/ethnic backgrounds, were conducted (one group with two Asians, seven Caucasians, and one African American and another group with five Asians and one African American). The first focus groups were conducted to describe customers' HIV-related risk behaviors and attitudes toward practicing safe sex with Asian masseuses in order to develop an intervention study. The second focus groups were conducted to describe customers' HIV-related risk behaviors and attitudes toward practicing safe sex with Asian masseuses, as well as to identify any changes in customers' attitudes and risk behaviors after the completion of the intervention study. In the intervention study, trained Korean and Vietnamese health educators provided eligible and consented Asian masseuses and massage parlor owners with three 1.5 hour individual counseling sessions to promote masseuses' healthy behaviors and safe sex with customers.

Each focus group lasted three hours. The inclusion/ exclusion criteria were as follows: (1) self-identified male, (2) 18 years or older, (3) being able to communicate in English, and (4) positive responses to both of the following questions: "Are you a regular customer at massage parlors?" and "Have you visited a massage parlor within the past 30 days?" A regular customer was defined as a person who visited massage parlors at least monthly. Potential participants were screened for eligibility when they called our project staff and screened again before participating in a focus group. The groups were moderated by trained Asian female project staff. The moderators read the informed consent form at the beginning of focus group, and consent was obtained from the participants. After the participants completed a brief survey, the moderators facilitated discussions using a discussion guide. The entire focus group discussion was digitally recorded and transcribed verbatim. Upon the completion of the focus group, participants were provided with monetary reimbursement and information about HIV/STIs testing services. The study protocol was approved by the Committee on Human Research, University of California, San Francisco.

Measures in the Short Survey. The short survey consisted of anchored questions about demographics and two measures that were modified from our previous study [3]: (1) AIDS knowledge, consisting of 20 questions, and (2) perceived norms toward practicing safe sex with masseuses (5-point Likert scale, from $1=$ strongly disagree to $5=$ strongly agree; Cronbach's alpha $=.76)$ (e.g., "Most of my friends think that I should always use a condom when I have sex with a masseuse").

Focus Group Discussion Guide. We followed the focus group discussion guide which covered the following areas: (1) issues and concerns in current life, (2) sexual behaviors with masseuses, (3) perception toward Asian masseuses and ideas about using massage parlors, (4) sexual behaviors with private 
partners, (5) HIV/STI knowledge, and (6) substance use behaviors.

Data Analysis. Qualitative analyses were guided based on grounded theory [20]. Three coders first read the focus group transcripts individually and identified emerging themes, following the discussion guide. Then, the themes were discussed by the coders together to develop a system of coding categories. Four major themes were identified in this study: (1) participants' sexual behaviors, (2) sexual behaviors under the influence of substances, (3) perception toward risks for HIV/STIs, and (4) perception toward Asian masseuses and massage parlors. Each coder directly entered assigned codes in the transcripts. Then, as a group, the coders discussed the discrepancies in coding and all members consented the coding.

2.2. Participants. A total of 34 male customers participated in the focus groups (47\% Asians, 32\% Caucasians, and $21 \%$ African Americans (see Table 1)). The mean age was 38 years old ( $\mathrm{SD}=10.3$, range $=21-68$ years). More than twothirds of the participants were single. Half of the participants had obtained college degrees or higher, and $44 \%$ of the participants earned more than $\$ 40,000$ per year. On average, participants frequented massage parlors twice a month (range = 1-8 times a month) and spent, on average, \$200 per visit (range $=\$ 65-\$ 1,000$ ), including admission $/$ massage fee and additional fees for sexual services. During the focus group discussions, three participants voluntarily revealed that they were infected with HIV, and 15 participants (44\%) reported having been infected with STIs in their lifetime (four reported gonorrhea, two genital herpes, one Hepatitis $\mathrm{C}$, and eight unspecified). Overall, there were no significant differences in the participants' demographic background between racial/ethnic groups, except for annual income; that is, Asian participants reported significantly higher income than other groups, $\chi^{2}(6, N=34)=13.9, P<.05$.

Because of the nature of focus group discussions, it is difficult to quantify or present the exact number or percentage of the participants who agree or disagree with certain statements or opinions. Therefore, the quantification of statements or opinions by terms such as "a few," "many," or "a majority" was based on our careful reading of the focus group transcript and the moderator's notes from observing discussions. In addition, the racial/ethnic background of the participants in the second focus group could not be identified because of the mixed racial/ethnic composition of the participants, except the participants' statements which explicitly indicated their racial/ethnic background.

\section{Results}

3.1. Condom Use at Massage Parlors. Focus group participants reported frequent visits to massage parlors and expressed having strong intentions to use condoms for vaginal sex with Asian masseuses due to fear of HIV/STIs. The participants also reported that condoms were usually
TABLE 1: Demographic characteristics $(n=34)$.

\begin{tabular}{lc}
\hline Variable & $\%$ \\
\hline Ethnicity & 47.1 \\
Asian & 20.6 \\
African-American & 32.4 \\
Caucasian & \\
Marital status & 67.6 \\
Single & 5.9 \\
Living with partner & 8.8 \\
Married & 11.8 \\
Divorced or separated & 5.9 \\
Widowed & \\
Highest level of education & 3.0 \\
Less than high school & 47.1 \\
High school graduate & 26.5 \\
College graduate & 23.5 \\
Graduate school & \\
Annual income & 23.5 \\
Less than $\$ 20,000$ & 32.4 \\
\$20,001 40,000 & 11.8 \\
\$40,001 50,000 & 32.4 \\
More than $\$ 50,000$ &
\end{tabular}

available at massage parlors and that masseuses initiated using condoms with them.

"They usually put it (a condom) on you....You do the shower or bath thing and then you lay down, and then they start rubbing you, and after you do the extra, like kissing on your neck, rubbing your leg, then they do their thing down there. Then, next thing you know the bitch (condom) is on. You know, most of them are pretty smooth by not breaking the moment."

Another echoed: "Some of them put it on with their mouth. Yeah, they are pretty smooth."

However, several participants expressed their preference to not use condoms.

"Basically, I had full (services)_oral sex, vagina sex, everything. No condoms or nothing. No condoms in the room ever. No condoms mentioned anything."

"Honestly, I've been HIV (positive) for three and a half years, but yeah, since that point (I started using condoms). But, before then, no (I did not use condoms). I'm not a really good condom fan."

Participants described instances when masseuses agreed to have unprotected sex with customers for extra money. However, none of the participants in the focus groups admitted to this behavior.

"And I really feel that the guys who come in there and he's gonna drop $\$ 400$ to $\$ 500$, and girl, he says 
I do not wanna use a condom, they gonna let them go without a condom. It depends on what you're spending."

"A friend of mine said that they use condoms during the first time and may be during the fifth visit, she will offer no to use it and she will say something like "I will offer this to you because you are special, you come and see (me) all the time. Do not tell anyone else."

Another participant followed stating that experienced masseuses did not negotiate extra money for not using a condom.

\begin{abstract}
"They aren't experienced as to what happens if they do not use it. However, it gets around pretty quick that if this girl is not using one that a lot of the clients stay away from (her) and she get a bad reputation because you are really taking a gamble on having unprotected sex with this person."
\end{abstract}

3.2. Infrequent Condom Use for Oral Sex. Unprotected oral sex was reported by a number of participants because they perceived that unprotected oral sex is not risky for HIV/STIs. Participants preferred having oral sex without using a condom unless masseuses insisted on using it.

"it seem oral sex with a condom, first of all, I think it's almost completely unnecessary."

"The only time I wouldn't (use condoms) would probably be oral sex, but if it's vaginal, definitely has to (use a condom)."

"And even oral, a lot of these girls are really conscious about HIV and they know it, they won't even give you oral without (using) a condom sometimes. But sometimes, I do not know, may be the girl is attracted to you and they feel safe or something and then they give you oral without (using) a condom."

3.3. Underestimating Risk and Susceptibility toward HIV/STIs. Participants perceived strong social norms toward practicing safe sex with masseuses ( $M=4.3$ on the measure). However, five and two participants reported having never been tested for STIs and HIV, respectively. The levels of AIDS-knowledge among the participants were low: they correctly answered 10 out of 20 questions, on average. Furthermore, the majority of participants reported low levels of perceived susceptibility toward HIV infection.

"I just do not have that fear of catching it (HIV). I can understand a woman getting it from a man, I can understand a man getting it from a man, and I can understand a drug user getting from each other. But I think the likelihood of me catching AIDS is very slim."
3.4. Reasons for Visiting Massage Parlors. Almost all participants cited solely having sexual intercourse with masseuses for sexual gratification or satisfaction without commitment or emotional connection as a reason for visiting massage parlors; "It's just the physical and with someone different than who you are going with or married to"; "you still have male urges, so you go to massage parlors, no strings attached, you just go there, do your businesses and leave."

Many participants also expressed that massage parlors were safe place for having sex with women. The secure environment of massage parlors with on-site presence of managers and security cameras made the participants not only feel that the establishments were safe and protected but also assume that masseuses were disease-free compared with street sex workers.

"Yeah, you feel like it's like a safe place to go (to a massage parlor). In my $30^{\prime} \mathrm{s}$,...I would like to go out to the street where there was more variety, more danger, and more excitement. And that fulfilled my fantasy...You know, people are getting violent, cops, getting busted. It's little more expensive (at massage parlors), but it's safe and cleaner."

"I won't go across the street anymore (to have sex with sex workers). I figure half of them are HIV positive. They've used drugs or get it from the needle. And, somebody at a massage parlor, they might be a little more safer."

"To me, it's a lot safer going to a massage parlor than picking up a girl on the street. If you pick up a girl on the street, you do have a chance of getting robbed, getting busted by police, getting embarrassed by going to jail, you know all that stuff."

3.5. Perception toward Asian Masseuses. Some participants expressed that they were looking for forbidden or exotic sexual encounters with Asian women, seeking intimacy, connection, and relationships and desiring to be treated well and respected. Participants also expressed their preference toward having sex with Asian women and biases and prejudices against Asian masseuses. Some participants expressed stereotypical views of Asian women as exotic, submissive, and docile, leading to sexual curiosity or fantasies about them.

"I was surprised the first time I went (to a massage parlor). I found that sex with Asian women can be very exciting."

"Being a young man from the South, there is something exotic about Asian women from my perspective. You know, so it satisfied a curiosity the first time."

"I like my woman to be somewhat I guess the submissiveness, the neatness is what I like. I will do a little role playing during sex. And, I will talk 
to them and say this is what I like, this is what I want you to be."

"I love my life, (but) there was just no sex there. So, for me, it was like that companionship, almost feeling like you are worthy or desirable. A better provider (Asian massage parlors) is going to make you feel that way."

Many participants expressed opinions that Asian masseuses might engage in sex work due to their limited English skills, immigration status, and economic needs. Some African-American and Caucasian participants expressed their attitudes to overpower and control masseuses and views about the business exchange with masseuses, which were fair to them.

"I really do not feel at all (toward masseuses). I know they are immigrants. Some of them do not want to be there. Some of them are trapped in the lifestyle or that's their life. And I figure, they're here. I am not going to be mean to them, (but) I might as well use them to satisfy my need."

"If it's just a paid thing (sex); then, it's just physical thing. Then, I have no real consideration for her (masseuse's) feelings. So, then, I am more apt to do what I want (her) to do for me, it is to get physical enjoyment as opposed to when I'm with someone I'm caring about."

Some Asian participants also acknowledged Asian masseuses' economic needs but tended to sympathize with the hardships of the masseuses, including the harsh working environment.

"I think they choose to be there. I think for whatever reason, they have family at home, they have to send money to (home), or they need to pay rent here,..., they come here thinking that they can find a job, and that's not going to pay rent. So,... whether they choose to be there or not, it's something I do not really think they like to do. I mean they may be very nice to you, but down deep inside, you know, they probably are 'oh it's just another client."

The customers acknowledged that they were paying not only for sex but also for being treated nicely by Asian masseuses. When masseuses challenged customers' stereotypical notion of Asian women as docile and obedient, the customers were stunned.

An African American stated: "I like to be in control, like to be pleased....And, I know in certain cultures, I am not an expert on Asian culture, I know that there are Chinese, Japanese, whatever, Thai. My perception is that it is a male dominated society. To me it's an added thrill and that's what I like."

"They (masseuses) do not have that street type mentality. They like hey, you here for a service and they treat you with kindness because they are going to get paid."
"Honestly, I think sex, selling sex is like necessary,...it's like a necessary evil. But, also looking at the position that these people are in, I think my actions are also kind of predatory. But it's kind like a power trip; ok, I paying you this amount of money you do this for me. I think they are necessary and sex will always be part of business in America."

A Caucasian man stated, "I do sometimes sorry for the girls (whom I did not pick). One girl kind of put up an argument, really got it was surprising. I expect the Asian woman to tend to play it or at least act submissive. And this girl started arguing....I was surprised."

3.6. Visiting Massage Parlors in San Francisco and Other Places. Many participants visited parlors multiple times in a month and some visited on a weekly basis. Some stated that they visited the same massage parlors for security reasons. Others stated that they preferred to see different nationalities of Asian masseuses or used websites to search for massage parlors that would satisfy their needs.

"I go [to a massage parlor] about twice a month, on average. Twice a month because I get paid every two weeks."

"It's like because I am a man, I go to massage parlors and I feel bad about it and do brag about with the fellows and...It's just sort of social thing more or less. So, it's really not a big deal, so I have been going (to massage parlors) since I was 21 years old."

"Usually I go to two particular favorites because those are the people that I trust. And they know me and I know them. They know what I want."

"If you just keep going to the same particular places all the time you see the same nationalities of people here. And, sometimes you just want to try and explore something else."

Several Asian participants revealed that they went to Asia for vacations and business trips and visited massage parlors on a daily basis during their trips.

"The culture like in Thailand, for example, sex is normal. Over here, there is a negative taboo on it. When I'm over there, everything is open, you know, sex is not big deal. So, there is less of taboo over there and much better selection (of women)."

Some participants talked about other sex venues in the USA, such as brothels in Nevada, where prostitution is legal in some counties.

"Now in Nevada, most of the places I've been in Nevada for a long time, you did not have to wear a condom. But now, they absolutely demand it. I mean that they've got condoms in every room. 
And, I was really disappointed 'cause I thought this one cat house was really good, 'cause they did not make the guys wear condoms. And then, 6 months later, they had stacks and stacks of condoms in every room. And girls were really careful on that. That was kind of a distraction."

3.7. Sex under the Influence of Alcohol and Drug Use. Many focus group participants described using alcohol and drugs before visiting massage parlors. Frequent alcohol use was commonly reported by Asian participants. Some Asians said that they would drink to release their tension and nervousness when visiting massage parlors. Other Asians reported often visiting massage parlors after heavy drinking with friends or business clients as a part of male-bonding or social activities. However, participants stated that massage parlors did not serve alcohol.

"The last time when I visited a massage parlor was probably when we had a bachelor party for one of my friends. And, (we) went around town and eventually had drinks. And, we ended up going to a massage parlor. It's an entertainment outlet."

"I take some (business) clients out (to massage parlors). I went (there) with some clients, and we were just out having a few drinks, chit chatting and one thing leads to another next thing you know, you end up there."

"They (massage parlors) do not serve beer there or anything, so I have never seen that, or anyone bringing in their own beer or anything like that."

African Americans and Caucasians revealed that they often visited a massage parlor while they were high on drugs. Many of them were currently using drugs, including two injection drug users (IDUs). Cocaine and marijuana were commonly used on a weekly basis, followed by methamphetamines/speed.

"There have been times that I was intoxicated (by drugs), you know, really messed up and the condom went out the window. So, you know, I have played some unsafe things."

"You know, the crystal meth It was a part of my addiction for a while because I had to do it everyday. I had to get high, get a call girl, go to a massage parlor every day."

3.8. HIV Prevention Programs. In spite of the participants' overall low levels of AIDS knowledge and perceived susceptibility toward HIV/STIs, some talked about the accessibility to HIV/STI prevention programs and their commitment to safe sex practices.

"I think in San Francisco, you know, we are uniquely qualified in the sense that because the AIDS epidemic pretty much sprouted here...that we have such good public service for STD and HIV testing....If you are willing to go and find it, and it's not hard."

"It's like wearing a seatbelt. I'm sorry. .. you do not even talk about it (condom). They have it. They are gonna put it on. It's a requirement."

Another participant responded, "Yeah, that's a good analogy actually. Would we agree prostitution should be legal, so long as it's illegal not to use condoms? Just as it's legal to drive, just (it's) not legal to drive without seatbelts."

Many participants stated that prostitution should be legalized to protect sex workers and customers from HIV /STIs.

"I think it should be legal because I mean if you are going to do it anyway, why not just make it legal instead of making people hide it."

"I think it should be legalized here, I mean many parts of the world, it is legal. It's run by the government, it's taxed, standard rates, they have health control, and even health insurance for the workers whether it's a man or woman."

\section{Discussion}

4.1. Summary. This study filled a gap in scientific knowledge about the context of massage parlors where male customers engage in sex with Asian masseuses. Previous studies identified the factors correlated with customers' unsafe sex with FSWs, such as negative attitudes toward sex workers and condom use, low levels of AIDS knowledge, and frequent use of sexual services $[10,14]$. Most of the previous HIV prevention studies targeted FSWs, not male customers, despite the fact that safe sex negotiation and safe or unsafe sex occur between customers and FSWs. The present study described male customers' sexual and drug use behaviors and attitudes toward practicing safe sex with Asian masseuses at massage parlors in San Francisco. The perception that the customers could engage in sex with masseuses without using a condom if they offered extra money was pervasive, although none of participants admitted this behavior. Customers' sexual behaviors with Asian masseuses were influenced by their perceived vulnerability toward HIV/STIs and substance use and by unspoken rules of condom use at parlors, as well as masseuses' initiation of condom use. Customers perceived massage parlors as a relatively safe place, compared to having sex with street sex workers. Many perceived that sex with masseuses was a simple exchange of money for sexual gratification without any emotional or social attachment with masseuses. They also showed cultural biases against Asian masseuses as docile and submissive. Some participants expressed an entitlement to receive requested sexual services from masseuses because they paid for the services. Findings of our current study have important implications for developing HIV prevention programs that would be applicable to "Asian" massage parlors in other large cities in the USA, as well as to massage parlors in Asian countries, such as China, Thailand, and Vietnam. 
4.2. Unsafe Sex between Customers and Masseuses. Our previous study showed that Asian masseuses had engaged in unsafe sex with customers in unique work environment of massage parlors in San Francisco [1, 2]. Strong economic incentives to earn extra money made many masseuses to succumb to their customers' requests to not use a condom. This situation was confirmed by the customers' comments that they could engage in sex with masseuses without using a condom if they paid extra money. Customers perceived that they could control the decision regarding condom use because of their economic advantage over masseuses and believed that they could receive their desired sexual services from masseuses for which they paid. However, a customer who was concerned about HIV/STIs encountered that masseuses who engaged in unsafe sex with customers would get a bad reputation and the customers who did wish to have safe sex would avoid the masseuses. Therefore, experienced or educated masseuses would not negotiate for extra money for unsafe sex. Focus group discussion revealed that customers used condoms with masseuses for vaginal sex most of the time and condoms were initiated by masseuses; however, customers still perceived that they could have sex with masseuses without using a condom because of their economic advantage over masseuses. Our data revealed that $11 \%$ of Asian masseuse participants did not use condoms for vaginal sex with customers and that self-efficacy, not economic pressure, was significantly and independently correlated with condom use with customers [21]. Further studies are needed to clarify who is willing to negotiate unsafe sex for extra money among customers and masseuses and what cultural and environmental factors (e.g., customer's perception toward and relationship with Asian masseuses and house rules of condom use) facilitate unsafe sexual behaviors at massage parlors.

4.3. Perception of Risk and HIV Prevention Programs. The other factor which had contributed to customers' decisions or preferences toward not using a condom with masseuses was customers' underestimation of risk for HIV/STIs based on their erroneous judgment about not having had STIs in the past. Maintaining these false assumptions seemed to correspond to a low prevalence of HIV/STI testing and low levels of HIV/AIDS knowledge, though it should be noted that some participants were very cautious to practice safe sex with masseuses and the majority perceived that massage parlors were safe places offering protection from HIV/STIs and arrests by police. The HIV testing rates in the USA are still low even among sexually active heterosexual males (e.g., $69 \%$ in 2002) [22]. Also, $42 \%$ of Asian masseuses in the intervention study reported that they had never been tested for HIV or that they had been tested but did not receive the results [21]. Some focus group participants commented on the San Francisco's historical HIV prevention efforts; however, prevention programs for Asian masseuses and male customers have not been prioritized by the local government due to low estimates of the HIV prevalence and incidence among Asian FSWs in the City and the budgetary constraints in HIV prevention programs. As the study for FSWs in
Philippines suggested [23], comprehensive HIV prevention programs that include promoting HIV/STI testing, providing health education for FSWs and managers, and enforcing $100 \%$ condom use policy at business venues need to be developed through addressing sociocultural and environmental factors specific to Asian massage parlors.

The focus group results clearly showed that the regulations and laws have changed customers' condom use behaviors. Participants recalled new regulations of condom use at brothels in Nevada. After the $100 \%$ condom use program was implemented in 1989 in Thailand, condom use among male customers at brothels reached over 90\% [24], and the rate of new HIV infections has dropped by $80 \%$ since 1991 [25]. Regulations such as $100 \%$ condom use at massage parlors in San Francisco and elsewhere should be considered as part of comprehensive harm reduction programs for FSWs using a multilevel approach [26]. In San Francisco, used or unused condoms cannot be presented as an evidence of prostitution to criminalize sex workers and customers according to the City's ordinance law [27]. Massage parlor managers should be educated regarding the City's law and advised to openly display condoms at the reception areas and massage rooms and explicitly state and enforce the house rules of $100 \%$ condom use.

4.4. Ethnic Group Differences, Substance Use, and Violence. Asian participants revealed the sociocultural context of visiting massage parlors as part of social bonding and business activities that were also reported in Asian countries, such as Vietnam [28] and China [29]. It is unknown whether customers at massage parlors in San Francisco play a role in bridging HIV/STIs to the general populations in the same mode as male customers in Asian countries. However, heavy drinking behaviors among Asian participants as part of their rituals of male-bonding or business would increase their risks for HIV/STIs. Our current study also revealed that some Caucasian and African-American participants reported using illicit drugs, such as marijuana, cocaine, and methamphetamines, before going to massage parlors to have sex with masseuses. As one participant described, methamphetamine use had driven him to seek sex at massage parlors. Some study participants believed that they could control the negotiation of sexual activities with masseuses and preferred not using a condom. One study participant described himself as a predator, but no one talked about violent behaviors against masseuses. Our intervention study showed that $28.6 \%$ and $13.5 \%$ of Asian masseuses had ever been exposed to verbal harassment and physical violence from customers, respectively [30]. Future studies need to investigate HIV risk behaviors in relation to customers' alcohol and illicit drug use and verbal and physical violence against masseuses.

4.5. Reasons for Having Sex with Asian Masseuses. Customers in the study perceived massage parlors as a safe environment. Victimization, such as assault, robbery, and rape, was more commonly reported among street sex workers than those working at establishments [31, 32]. Call girls, escorts, and 
massage parlor workers were able to screen out customers and have regular customers [33]. Nevada's legal brothels provide sex workers with protections such as safety devices and minimize HIV/STI risks for sex workers and customers. There were no HIV cases reported among registered FSWs in Nevada's legal brothels since the mandatory testing was implemented in the mid 1980's [34]. One participant expressed an analogy of a law requiring seatbelt for driving a car and condom use with sex workers if prostitution is legalized.

Customers are diverse in terms of age, race, class, and marital status; however, they patronize sex workers for similar reasons, such as a desire for certain types of sexual acts, image of women, and specific physical features (e.g., exotic Asian women), avoiding emotional attachment or connection and difficulty in finding women through conventional ways [35]. Study participants expressed similar reasons for frequenting massage parlors, though some African-Americans and Caucasians expressed stereotypical views about Asian women and a sense of entitlement to have optimal sexual services from Asian masseuses. Male customers often desire emotional support and companionship from call girls and escort services and less so from street sex workers [33]. A few study participants expressed a desire to receive emotional support and intimacy from Asian masseuses. The emotional or personal relationships between a masseuse and a regular customer would create a risky situation for HIV/STIs. Further research is needed to understand the process in which customers and masseuses perceive the relationship as "regular" and engage in unprotected sex.

4.6. Limitations of the Study. Extrapolation of the study results to other massage parlors in San Francisco or elsewhere must be made with caution because participants were recruited using purposive sampling in two different time periods separated by four years. Also, participants' statements or opinions might not represent those of male customers that frequented Asian massage parlors in San Francisco. In addition, focus group discussions might be biased because of social desirability.

4.7. Recommendations. Immigrant Asian masseuses and massage parlors in San Francisco have been featured by television documentary and news articles [36, 37]. Media coverage tended to portray Asian masseuses as trafficked from Asian countries and forced to work as sex workers (often described as "sex slaves") at harsh work environments with little hope of escaping the situations. As it may be intended, media attention often provokes elected officials and law enforcement officers to voice their commitment against prostitution and trafficking without addressing a long term solution to the "problems" and public health issues surrounding sex work businesses. Comprehensive HIV/STI prevention programs for FSWs must be implemented at multiple levels: individual (e.g., AIDS education, skills training for negating safe sex and financial management, and developing skills for alternative jobs), work place/venues (e.g., enforcing 100\% condom use policy, creating support groups for sex workers, developing norms toward practicing safe sex, and facilitating medical check-up and STI testing), and community, local, and national levels (e.g., increase the access to and utilization of health care and HIV/STI testing, promoting human rights for sex workers, and implementation of $100 \%$ condom use policy and other harm reduction policies).

\section{Conflict of Interests}

The authors declare that there is no conflict of interests regarding the publication of this paper.

\section{Acknowledgments}

This study was supported by the National Institute on Drug Abuse (NIDA) and the National Institute of Health (PI: Tooru Nemoto, Grant no. R01DA13896). The authors thank Samantha Witt, Alefiyah Pishori, Mary Hsueh, Tracy Tam, and other project staff and study participants. Part of the study results were presented at the International AIDS Conference in 2004 and the American Public Health Association's annual meeting in 2004 and 2007. The opinions and recommendations expressed in this paper are solely those of the authors and do not necessarily represent the views of NIDA.

\section{References}

[1] T. Nemoto, M. Iwamoto, H. J. Oh, S. Wong, and H. Nguyen, "Risk behaviors among Asian women who work at massage parlors in San Francisco: perspectives from masseuses and owners/managers," AIDS Education and Prevention, vol. 17, no. 5, pp. 444-456, 2005.

[2] T. Nemoto, M. Iwamoto, S. Wong, N. L. Mai, and D. Operario, "Social factors related to risk for violence and sexually transmitted infections/HIV among Asian massage parlor workers in San Francisco," AIDS and Behavior, vol. 8, no. 4, pp. 475-483, 2004.

[3] T. Nemoto, D. Operario, M. Takenaka, M. Iwamoto, and M. N. Le, "HIV risk among Asian women working at massage parlors in San Francisco," AIDS Education and Prevention, vol. 15, no. 3, pp. 245-256, 2003.

[4] B. Ki-moon, Redefining AIDS in Asia-Crafting An Effective Response, Report of the Commission on AIDS in Asia, New Delhi, India, 2008.

[5] Monitoring the AIDS Pandemic, "AIDS in Asia: Face the Facts-A Comprehensive Analysis of the AIDS Epidemic in Asia," 2004, http://aidsdatahub.org/dmdocuments/AIDS_in_ Asia_Face_the_Facts_2004.pdf.

[6] The World Bank, "The World Bank's Commitment to HIV/AIDS in AFRICA, our Agenda for Action, 2007-2011," 2008, http://siteresources.worldbank.org/INTAFRREGTOPHFVAIDS/Resources/WB_HIV-AIDS-AFA_2007-2011_Advance_ Copy.pdf.

[7] M. E. Gomes Do Espirito Santo and G. D. Etheredge, "Male clients of brothel prostitutes as a bridge for HIV infection between high risk and low risk groups of women in Senegal," Sexually Transmitted Infections, vol. 81, no. 4, pp. 342-344, 2005.

[8] M. L. Wong, R. K. W. Chan, D. Koh, M. E. Barrett, S. K. Chew, and S. S. H. Wee, "A comparative study of condom use and self-reported sexually transmitted infections between foreign 
Asian and local clients of sex workers in Singapore," Sexually Transmitted Diseases, vol. 32, no. 7, pp. 439-445, 2005.

[9] R.-S. Luan, Y.-L. Zeng, L. Fan et al., "A study on acquired immune deficiency syndrome related behaviors in male clients of female sex workers," Zhonghua Liu Xing Bing Xue Za Zhi, vol. 26, no. 2, pp. 101-105, 2005.

[10] B. Wang, X. Li, B. Stanton, X. Fang, D. Lin, and R. Mao, "HIV-related risk behaviors and history of sexually transmitted diseases among male migrants who patronize commercial sex in China," Sexually Transmitted Diseases, vol. 34, no. 1, pp. 1-8, 2007.

[11] J. T. F. Lau and H. Y. Tsui, "Behavioral surveillance surveys of the male clients of female sex workers in Hong Kong: results of three population-based surveys," Sexually Transmitted Diseases, vol. 30, no. 8, pp. 620-628, 2003.

[12] H. Ward, C. H. Mercer, K. Wellings et al., "Who pays for sex? An analysis of the increasing prevalence of female commercial sex contacts among men in Britain," Sexually Transmitted Infections, vol. 81, no. 6, pp. 467-471, 2005.

[13] N. T. T. Thuy, C. P. Lindan, T. H. Phong et al., "Predictors of visits to commercial sex workers by male attendees at sexually transmitted disease clinics in southern Vietnam," AIDS, vol. 13, no. 6, pp. 719-725, 1999.

[14] S. Wee, M. E. Barrett, W. M. Lian, T. Jayabaskar, and K. W. R. Chan, "Determinants of inconsistent condom use with female sex workers among men attending the STD clinic in Singapore," Sexually Transmitted Infections, vol. 80, no. 4, pp. 310-314, 2004.

[15] J. T. F. Lau, P. C. Siah, and H. Y. Tsui, "Behavioral surveillance and factors associated with condom use and STD incidences among the male commercial sex client population in Hong Kong-results of two surveys," AIDS Education and Prevention, vol. 14, no. 4, pp. 306-317, 2002.

[16] N. M. Thang, V. T. Huong, and M.-E. Blanc, "Sexual behaviour related to HIV/AIDS: commercial sex and condom use in Hanoi, Viet Nam," Asia-Pacific Population Journal, vol. 17, no. 3, pp. 41-52, 2002.

[17] R. Weitzer, "Why we need more research on sex work," in Sex For Sale: Prostitution, Pornography, and the Sex Industry, R. Weitzer, Ed., pp. 1-16, Routledge, New York, NY, USA, 2000.

[18] K. W. Elifson, J. Boles, W. W. Darrow, and C. E. Sterk, "HIV seroprevalence and risk factors among clients of female and male prostitutes," Journal of Acquired Immune Deficiency Syndromes and Human Retrovirology, vol. 20, no. 2, pp. 195-200, 1999.

[19] A. E. Albert, D. L. Warner, and R. A. Hatcher, "Facilitating condom use with clients during commercial sex in Nevada's legal brothels," American Journal of Public Health, vol. 88, no. 4, pp. 643-646, 1998.

[20] B. G. Glaser and A. L. Strauss, The Discovery of Grounded Theory: Strategies For Qualitative Research, Aldine De Gruyter, New York, NY, USA, 1967.

[21] T. Nemoto, M. Iwamoto, D. Tran et al., "Individual and system level health promotion project for Asian female sex workers in San Francisco, U.S.A.," in Proceedings of the International AIDS Conference, Toronto, Canada, 2006.

[22] Centers for Disease Control and Prevention, HIV Testing Survey, 2002, U.S. Department of Health and Human Services, Centers for Disease Control and Prevention, Atlanta, Ga, USA, 2004.

[23] D. E. Morisky, M. Peña, T. V. Tiglao, and K. Y. Liu, "The impact of the work environment on condom use among female bar workers in the Philippines," Health Education and Behavior, vol. 29, no. 4, pp. 461-472, 2002.

[24] UNAIDS, Evaluation of the 100\% Condom Programme in Thailand, UNAIDS, Geneva, Switzerland, 2000.

[25] Thai Working Group on HIV/AIDS Projection, Projections For HIV/AIDS in Thailand: 2000-2020, AIDS Division, Department of Communicable Disease Control, Ministry of Public Health, Bangkok, Thailand, 2001.

[26] M. L. Rekart, "Sex-work harm reduction," The Lancet, vol. 366, no. 9503, pp. 2123-2134, 2005.

[27] Board of Supervisors of the City and County of San Francisco, California, "The San Francisco Task Force on ProstitutionFinal Report," 1996, http://www.bayswan.org/4laws.html.

[28] H. M. Phinney, "Rice is essential but tiresome; You should get some noodles': Doi Moi and the political economy of men's extramarital sexual relations and marital HIV risk in Hanoi, Vietnam," American Journal of Public Health, vol. 98, no. 4, pp. 650-660, 2008.

[29] E. Uretsky, “Mobile men with money': the socio-cultural and politicoeconomic context of "high-risk" behaviour among wealthy businessmen and government officials in urban China," Culture, Health and Sexuality, vol. 10, no. 8, pp. 801-814, 2008.

[30] T. Nemoto, M. Iwamoto, M. Sakata, and E. Eilkhani, "HIVRelated Risk Behaviors and Exposure to Violence among Asian Women Who Work at Massage Parlors in San Francisco (unpublished paper-under review)," 2013.

[31] R. Perkins and F. Lovejoy, "Healthy and unhealthy life styles of female brothel workers and call girls (private sex workers) in Sydney," Australian and New Zealand Journal of Public Health, vol. 20, no. 5, pp. 512-516, 1996.

[32] D. Whittaker and G. Hart, "Manging risks: the social organization of indoor prostitution," Sociology of Health and Illness, vol. 18, pp. 399-414, 1996.

[33] J. Lever and D. Dolnick, Clients and Call Girls: Seeking Sex and Intimacy, Routledge, New York, NY, USA, 2000.

[34] Overdrive, "Nevada Brothels," 2002, http://www.overdriveonline.com/nevada-brothels/?pg=2.

[35] R. Weitzer, "New directions in research on prostitution," Crime, Law and Social Change, vol. 43, no. 4-5, pp. 211-235, 2005.

[36] M. Viera, "MSNBC Undercover: Sex Slaves in America," 2008, http://www.msnbc.msn.com/id/22056066/ns/msnbc_tvdocumentaries/t/msnbc-undercover-sex-slaves-america/.

[37] M. May, "San Francisco Is A Major Center For International Crime Networks That Smuggle And Enslave," San Francisco Chronicle, 2006, http://www.sfgate.com/cgi-bin/article.cgi?f=/ c/a/2006/10/06/MNGR1LGUQ41.DTL\#ixzzlahXWKLlC. 


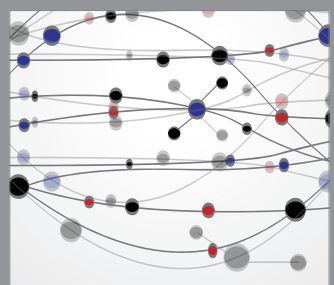

The Scientific World Journal
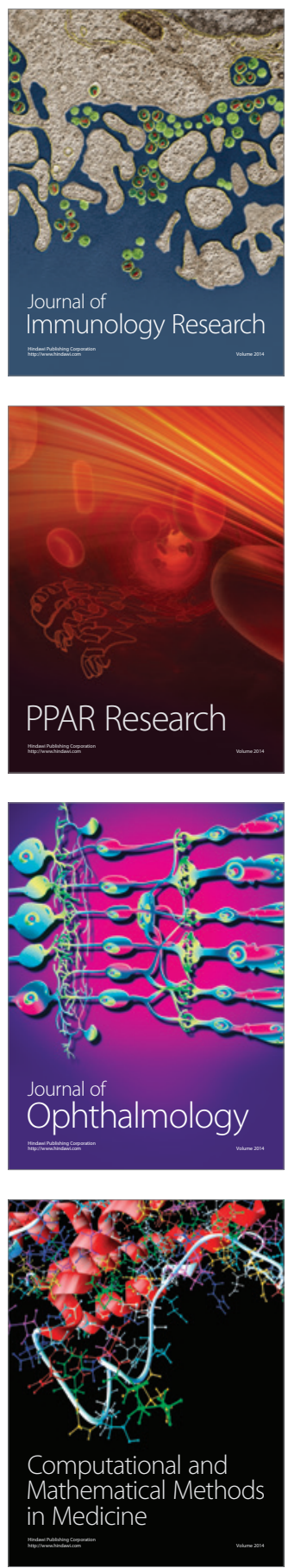

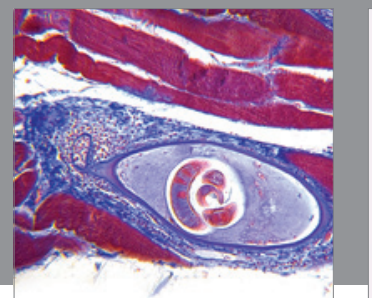

Gastroenterology

Research and Practice
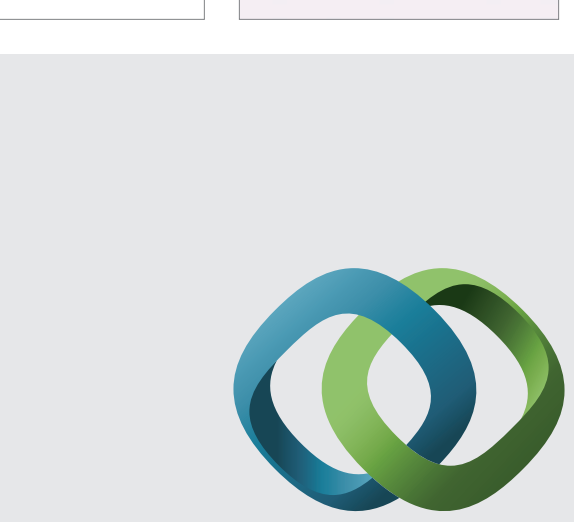

\section{Hindawi}

Submit your manuscripts at

http://www.hindawi.com
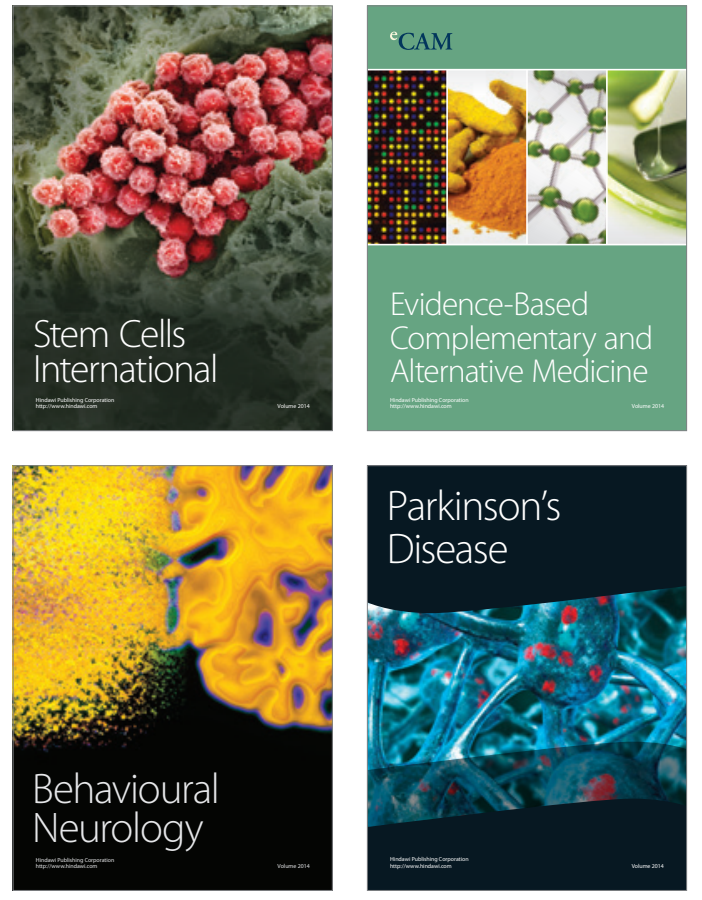
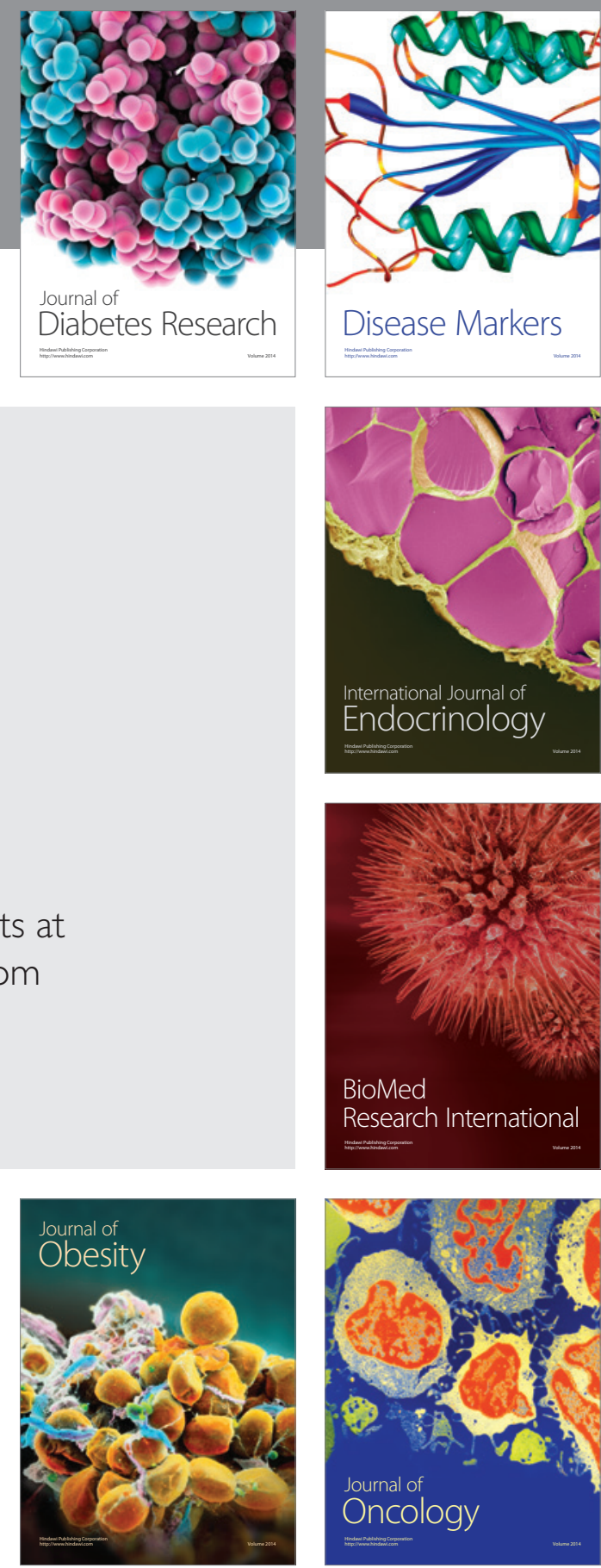

Disease Markers
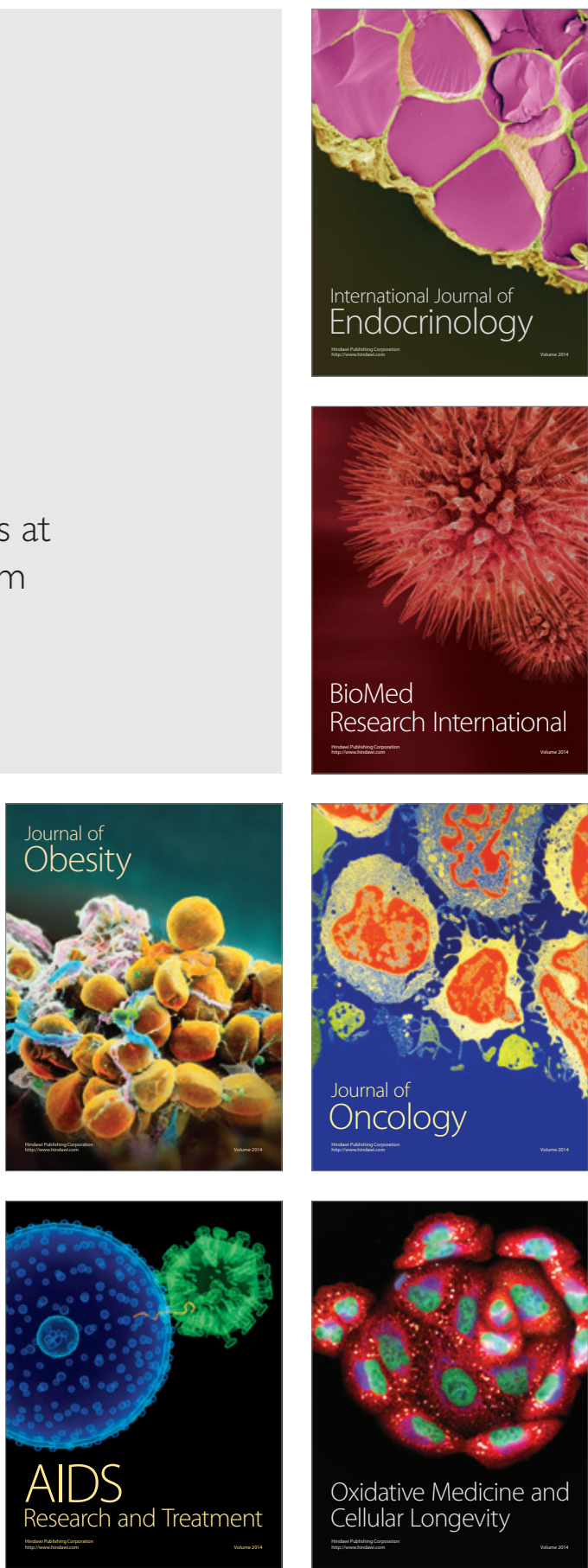